\title{
Use of HfC(310) as a High Brightness Electron Sources for Advanced Imaging Applications.
}

\author{
J.M. Lovell ${ }^{1}$, W.A. Mackie ${ }^{1,2}$, T.W. Curtis ${ }^{1}$, and G.G. Magera ${ }^{1}$. \\ 1. Applied Physics Technologies, Inc. 1600 NE Miller St., McMinnville, OR, 97128, USA. \\ 2. Linfield Research Institute, 900 SE Baker St., McMinnville, OR 97128, USA.
}

This paper continues our work to demonstrate a new electron source for applications where high brightness is required. Thermionic emission and cold field emission are ends in a continuum of electron emission processes; between lays extended Schottky emission (ESE) and thermal-field emission (TFE). Presently, commercial electron sources operate in the extended Schottky or cold field emission (CFE) modes both using tungsten as the base material. It is known that surface tension and field forces contribute to blunting or build-up on these W based emitters. However, HfC sources have activation energy for surface migration much large than for W. This coupled with loosely bound surface contaminants mean operation at elevated temperatures can keep the surface clean but not trigger geometric changes.

Transition metal carbide emitters have very high current capability, can be tolerant of moderate vacuum, and are capable of stable operation over a large temperature range. $\mathrm{HfC}(310)$ provides a relatively low work function $(\sim 3.4 \mathrm{eV})$, has a low evaporation rate[1], is resistant to ion bombardment and sputtering, has a high melting point ( $>4000 \mathrm{~K})$, and a low surface mobility.

Both modeling and experimental performance are reported for $\mathrm{HfC}(310)$ cathodes where emission is studied over a range of temperatures to $\sim 2000 \mathrm{~K}$ which covers TFE and SE modes. Reduced brightness, energy spread, and stability values were obtained in CFE operation with energy spread $(\sim 310 \mathrm{meV})$ lower by a factor of two and reduced brightness $\left(\sim 3 \times 10^{8} \mathrm{~A} / \mathrm{m}^{2} / \mathrm{sr} / \mathrm{V}\right)$ higher by a factor of five than a $\mathrm{ZrO} / \mathrm{W}$ Schottky source. However, oper $_{2}$ ation in extended Schottky mode resulted in electron optical reduced brightness levels to $\sim 8 \times 10^{9} \mathrm{~A} / \mathrm{m}^{2} / \mathrm{sr} / \mathrm{V}$, roughly 10-100x higher than commercial Schottky sources. We use $B_{r}=I^{\prime} /\left(\pi r_{v}{ }^{2} V_{E}\right)$ to calculate reduced brightness where I' is angular intensity, $r_{v}$ is the virtual source radius obtained from modeling, and $\mathrm{V}_{\mathrm{E}}$ is the beam voltage.

In terms of angular intensity, HfC sources are capable of higher levels than commercial $\mathrm{ZrO} / \mathrm{W}$ Schottky sources due to the nature of the material. $\mathrm{ZrO} / \mathrm{W}$ sources require a balance of electric field and temperature to keep the $\mathrm{W}$-substrate end-form constant. This field/temperature balance is also needed in the supply of $\mathrm{ZrO}$ to the apex but puts limits on the angular intensity. HfC sources have no need for a material supply and because of their robustness can be operated at high temperatures and high fields thereby not limiting the angular intensity to a relatively small range. Generally the maximum value of I' for $\mathrm{ZrO} / \mathrm{W}$ sources is $\sim 1 \mathrm{~mA} / \mathrm{sr}$ [2] whereas HfC sources have been operated to $>60 \mathrm{~mA} / \mathrm{sr}$.

HfC(310) emitters with several end-form geometries were operated in a Philips XL40 FEG SEM and compared with $\mathrm{ZrO} / \mathrm{W}(100)$ emitters operated under similar conditions. Several improvements were noted during operation due primarily to the end-form geometrical differences. For example we compared a $\mathrm{ZrO} / \mathrm{W}$ emitter with a facet diameter of $\sim 300 \mathrm{~nm}$ to a $\mathrm{HfC}$ emitter with a rounded end-form radius of $\sim 220 \mathrm{~nm}$. 
We were able to obtain much higher beam currents with $\mathrm{HfC}$ as compared to $\mathrm{ZrO} / \mathrm{W}$ sources for identical SEM operating conditions. Fig. 1 compares Faraday cup measured beam currents using the XL40's larger $1200 \mu \mathrm{m}$ aperture. The extraction voltage range differed slightly; the ranges shown in the figure were $3.5-4.5 \mathrm{kV}$ and $4.0-5.0 \mathrm{kV}$ for the $\mathrm{ZrO} / \mathrm{W}$ and $\mathrm{HfC}$ sources respectively. Note too that the column current is directly proportional to the average angular intensity of the source since the drift tube of the XL40 collects emission prior to the beam limiting aperture; here $\sim 8 \mu \mathrm{A}$ is roughly equal to $\sim 0.5$ $\mathrm{mA} / \mathrm{sr}$. The higher axial current delivered by the HfC source is a function of the emission distribution which peaks axially whereas the $\mathrm{ZrO} / \mathrm{W}$ source has a flat distribution surrounding the axial direction. [2]

Beam current fluctuations over time were also measured in the XL40. Faraday cup measured beam currents we collected over time where we observed standard deviation values of $\sim 0.04 \%$ and $\sim 0.07 \%$ for $\mathrm{ZrO} / \mathrm{W}$ and $\mathrm{HfC}$ respectively.

There are certainly several differences between $\mathrm{ZrO} / \mathrm{W}$ and $\mathrm{HfC}$ operation. Specifically the nature of the materials necessitates different emitter crystal mount methods; spot welding vs. the Vogel mount[3]. More heater power was needed for the HfC and more heat was generated in the gun which ideally needs to be reduced. Optimization of gun geometry is presently being addressed along with operation under different SEM settings. Rounded vs. truncated emitters are also being explored further.

However, with this work we have documented the potential for the $\mathrm{HfC}(310)$ source operated in extended Schottky mode. Broader angular intensities are possible since these sources are not dependent upon a supply function of $\mathrm{Zr} / \mathrm{O}$ and hence can operate over a larger range of temperatures, fields, and pressures. Of greater importance is the potential for higher electron optical brightness which is due primarily to the ability to use the rounded emitter end-form in the Schottky emission regime.[4]

[1] W.A. Mackie and P.R. Davis, IEEE Trans. Electron Devices, 36, 220 (1989).

[2] L.W. Swanson and G.A. Schwind, in Handbook of Charged Particle Optics, edited by J. Orloff (CRC, Boca Raton, FL, 1997), Chap. 2, pp. 77-102.

[3] www.a-p-tech.com.

[4] The authors acknowledge that financial support was provided in part by Air Force Research Laboratory (AFRL). Joe Hancock is thanked for electronic and vacuum system support.

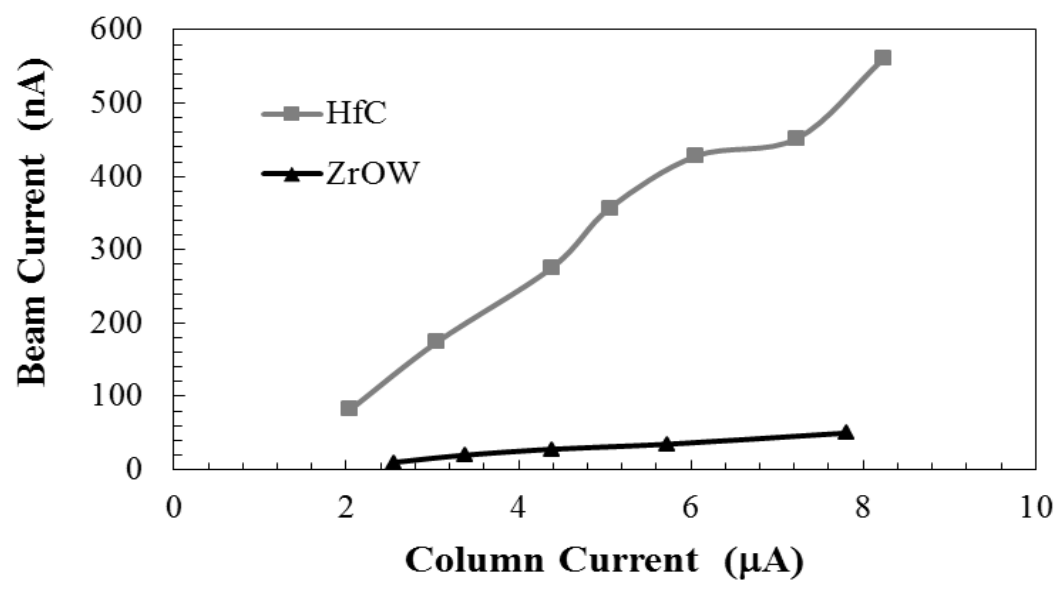

Figure 1. Measured beam currents compared using the $1200 \mathrm{~mm}$ aperture and identical SEM settings. 